QCD Evolution Workshop 2014

International Journal of Modern Physics: Conference Series

Vol. 37 (2015) 1560049 (8 pages)

(C) The Author

DOI: $10.1142 / \mathrm{S} 2010194515600496$

\title{
Joint Resummation for TMD Wave Function of Pion
}

\author{
Yu-Ming Wang \\ Physik Department T31, Technische Universität München, \\ James-Franck-Straße 1, D-85748 Garching, Germany \\ Institut für Theoretische Teilchenphysik und Kosmologie, \\ RWTH Aachen University, D-52056 Aachen, Germany \\ yuming.wang@tum.de
}

Published 25 February 2015

\begin{abstract}
QCD corrections to transverse-momentum-dependent pion wave function develop the mixed double logarithm $\ln x \ln \left(\zeta_{P}^{2} / k_{T}^{2}\right)$, when the gluon emission is collinear to the energetic pion. The fist scheme-independent $k_{T}$ factorization formula for $\gamma^{*} \pi \rightarrow \gamma$ transition form factor is achieved by resumming all the enhanced logarithms for both pion wave function and short-distance coefficient function. High-order QCD corrections and transfer momentum $\sqrt{Q^{2}}$ dependence of pion form factor are found to be distinct from those predicted by the conventional resummation approach.
\end{abstract}

Keywords: Resummation; QCD; electromagnetic processes and properties.

PACS numbers: 11.10.Hi, 12.38.-t, 13.40.-f

\section{Introduction}

Transverse-momentum-dependent (TMD) wave functions serve as fundamental ingredients of calculating various hard exclusive processes in the framework of $k_{T}$ factorization. Stimulated by the so-called "scaling violation" of $\gamma^{*} \pi \rightarrow \gamma$ form factor from BaBar measurement, ${ }^{1}$ intensive efforts have been devoted to the studies of light-cone distribution amplitude (LCDA) and TMD wave function of pion, including many speculations about the the end-point behavior of pion LCDA. It was argued in Ref. [2] that power suppressed soft corrections characterized by the longdistance photon radiation and end-point contribution, computed from light-cone QCD sum rules, can induce the scaling violation at moderate momentum transfer, without resorting to the non-vanishing pion LCDA at the end points. ${ }^{3}$ Applying the $k_{T}$ factorization approach, a reasonable description of BaBar was achieved ${ }^{4}$ after taking into account of the $Q^{2}$ dependence of threshold resummation for the double logarithm $\ln ^{2} x$. However, the conventional resummation techniques, organizing

This is an Open Access article published by World Scientific Publishing Company. It is distributed under the terms of the Creative Commons Attribution 3.0 (CC-BY) License. Further distribution of this work is permitted, provided the original work is properly cited. 
the Sudakov and threshold logarithms in TMD factorization, leave out the single rapidity logarithm $\ln \left(\zeta^{2} / k_{T}^{2}\right)$ and the mixed logarithm $\ln x \ln \left(\zeta^{2} / k_{T}^{2}\right)$ involved in the next-to-leading-order (NLO) QCD corrections to pion wave function ${ }^{5-7}$ due to the regularization of light-cone divergence. ${ }^{\text {a }}$ Resummation of the mixed and single rapidity logarithms, going beyond Ref. [4], is essential to achieve a scheme independent TMD factorization formula which is desirable to discern whether the BaBar anomaly reveals an unusual behavior of pion wave function.

As both the $x$ and $k_{T}$ dependencies of TMD pion wave function are distorted by the resummation of mixed logarithm $\ln x \ln \left(\zeta^{2} / k_{T}^{2}\right)$, a simultaneous treatment of small $x$ and Sudakov enhancements is therefore in demand, calling for the joint resummation technique developed in Ref. [11]. It is the aim of Ref. [12] to construct the evolution equations for TMD pion wave function and hard scattering kernel of $\gamma^{*} \pi \rightarrow \gamma$ transition removing the factorization-scheme dependence for the first time. This approach is also sufficiently flexible to be applied in more complex hard reactions such as electromagnetic pion form factor, for which the evolution equations for the TMD wave functions of both initial and final state hadrons are needed.

\section{Rapidity Evolution Equation}

In contrast to the pion LCDA in collinear factorization, the definition of TMD pion wave function is more involved ${ }^{12}$

$$
\begin{aligned}
\Phi\left(x, k_{T}, \zeta^{2}, \mu_{f}\right)= & \int \frac{d y^{+}}{2 \pi} \frac{d^{2} y_{T}}{(2 \pi)^{2}} e^{-i x P^{-} y^{+}+i \mathbf{k}_{T} \cdot \mathbf{y}_{T}} \\
& \times\left\langle 0\left|\bar{q}(y) W_{y}(u)^{\dagger} I_{u ; y, 0} W_{0}(u) \not_{+} \gamma_{5} q(0)\right| \pi(P)\right\rangle,
\end{aligned}
$$

following Ref. [13], where the quark coordinate $y=\left(y^{+}, 0, \mathbf{y}_{T}\right)$ is off the light cone and the rapidity parameter is defined as $\zeta^{2}=4\left(n_{-} \cdot u\right)^{2} / u^{2}$. Proposing a justified TMD wave function or parton density to be used in the $k_{T}$ factorization formula is under rapidly progress recently and a critical overview of various definitions in both QCD and SCET can be found in Ref. [14]. Nevertheless, it is worthwhile to point out that the Wilson line self-energy corrections in the above definition can induce divergences proportional to the length of Wilson line. ${ }^{15}$ To get rid of such divergences, one can either introduce the soft subtraction factor to cancel that from the unsubtracted TMD parton density as suggested in Ref. [16] or design the Wilsonline structure in the non-local matrix element to facilitate the soft subtraction. ${ }^{17}$ In the following, I will take the definition present in Eq. (1) as an illustrative example to elaborate the strategy of performing the joint resummation and the application of this technique to the modified definition of TMD pion wave function constructed in Ref. [17] is straightforward.

\footnotetext{
${ }^{a}$ The double rapidity logarithm $\ln ^{2}\left(\zeta^{2} / k_{T}^{2}\right)$ appeared in the NLO $B$-meson wave function ${ }^{8-10}$ does not emerge in the pion case because of the cancellation of soft enhancement.
} 


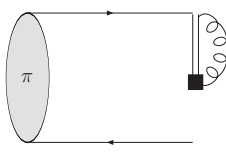

(a)



)

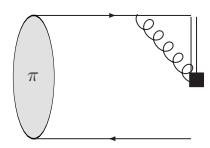

(b)

Fig. 1. Rapidity evolution kernel of the TMD pion wave function contributed from soft gluon exchange (diagram (a)) and hard gluon radiation (diagram (b)).

The starting point to derive the evolution equation is trading the rapidity derivative to the variation of the gauge vector $u$ defining the Wilson lines

$$
\zeta^{2} \frac{d}{d \zeta^{2}} \Phi=-\frac{u^{2}}{n_{-} \cdot u} \frac{n_{-}^{\alpha}}{2} \frac{d}{d u^{\alpha}} \Phi,
$$

due to the obvious advantage that the $u$-dependence can only arise from the Wilson line interactions. Rapidity derivative of the Feynman rule associated with the Wilson line is then given by

$$
\zeta^{2} \frac{d}{d \zeta^{2}} \frac{u^{\beta}}{u \cdot l+i \epsilon}=\frac{\hat{u}^{\beta}}{2 u \cdot l}
$$

where the special vertex

$$
\hat{u}^{\beta}=\frac{u^{2}}{n_{-} \cdot u}\left(\frac{n_{-} \cdot l}{u \cdot l} u^{\beta}-n_{-}^{\beta}\right)
$$

will suppress the collinear gluon emission from the Wilson link. The evaluation of rapidity evolution kernel can be obtained by computing the soft and hard gluon radiation effects as displayed in figure 1, following the reasonings in Ref. [18].

Using the Feynman rule present in Eq. (3), one can easily write down the soft and hard gluon contributions (i.e., the well-known $K$ and $G$ functions)

$$
\begin{aligned}
K_{1}= & -\frac{i g^{2} C_{F}}{2} \int \frac{d^{4} l}{(2 \pi)^{4}} \frac{\hat{u} \cdot n_{-}}{(u \cdot l+i \epsilon)\left(l^{2}+i \epsilon\right)\left(n_{-} \cdot l+i \epsilon\right)}, \\
K_{2} \otimes \Phi= & \frac{i g^{2} C_{F}}{2} \int \frac{d^{4} l}{(2 \pi)^{4}} \frac{\hat{u} \cdot n_{-}}{(u \cdot l+i \epsilon)\left(l^{2}+i \epsilon\right)\left(n_{-} \cdot l+i \epsilon\right)} \\
& \times \Phi\left(x-l^{-} / P^{-},\left|\mathbf{k}_{T}-\mathbf{l}_{T}\right|, \zeta^{2}, \mu_{f}\right),
\end{aligned}
$$

for the $K$ function, and

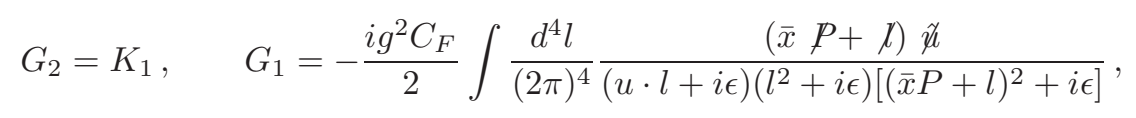

for the $G$ function with the variable $\bar{x} \equiv 1-x$. A straightforward calculation of the contribution from soft reducible diagram yields

$$
K_{1}=-\frac{\alpha_{s} C_{F}}{4 \pi}\left(\frac{1}{\hat{\epsilon}}+\ln \frac{\mu_{f}^{2}}{\lambda^{2}}\right),
$$

with $1 / \hat{\epsilon} \equiv 1 / \epsilon-\gamma_{E}+\ln 4 \pi$, where the gluon mass $\lambda$ is introduced to regularize the infrared divergence to be cancelled by that from the soft irreducible contribution 
$K_{2} \otimes \Phi$. The ultraviolet scale dependence of soft $K$ function will cancel against the one from $G$ function, as the mixed logarithm $\ln x \ln \left(\zeta^{2} / k_{T}^{2}\right)$ to be resummed is independent of renormalization/factorization scale. To compute the irreducible contribution $K_{2} \otimes \Phi$, one can apply the joint Mellin and Fourier transformations for TMD wave function, and factorize the evolution kernel

$$
\tilde{K}_{2}=\frac{\alpha_{s} C_{F}}{2 \pi}\left[K_{0}(\lambda b)-K_{0}\left(\frac{\zeta P^{-} b}{N}\right)\right],
$$

where the subleading power corrections $1 / \zeta^{2 n}(n \geq 1)$ are neglected. Adding up the two pieces together, the bare soft function is given by

$$
\tilde{K}^{(b)}=K_{1}+\tilde{K}_{2}=-\frac{\alpha_{s} C_{F}}{4 \pi}\left(\frac{1}{\hat{\epsilon}}+\ln \frac{\mu_{f}^{2} N^{2}}{\zeta^{2} P^{-2}}\right),
$$

in the limit $N \gg \zeta P^{-} b$, which is the soft function appearing in the threshold resummation, and

$$
\tilde{K}^{(b)}=K_{1}+\tilde{K}_{2}=-\frac{\alpha_{s} C_{F}}{2 \pi}\left[\frac{1}{2 \hat{\epsilon}}+\ln \left(\mu_{f} b\right)+\gamma_{E}-\ln 2\right],
$$

for $N \ll \zeta P^{-} b$, which is the soft function entering the $k_{T}$ resummation. It is clear that the unification of the threshold and $k_{T}$ resummations is achieved simultaneously. In what follows, we will work with the $N \gg \zeta P^{-} b$ limit for the sake of simplifying the analysis of inverse Mellin transformation which is essential to transform the TMD wave function into the usual momentum space.

Computing the bare $G$-function in the same way yields

$$
G^{(b)}=G_{1}-G_{2}=\frac{\alpha_{s} C_{F}}{4 \pi}\left[\frac{1}{\hat{\epsilon}}+\ln \frac{\mu_{f}^{2}}{\zeta^{2}\left(\bar{x} P^{-}\right)^{2}}-4\right] .
$$

Implementing the ultraviolet subtraction and including the renormalization-group $(\mathrm{RG})$ improvement lead to the rapidity evolution kernel

$$
\tilde{K}^{(r)}\left(\mu_{f}\right)+G^{(r)}\left(\mu_{f}\right)=\tilde{K}^{(r)}\left(\mu_{0}\right)+G^{(r)}\left(\mu_{1}\right)-\int_{\mu_{0}}^{\mu_{1}} \frac{d \tilde{\mu}}{\tilde{\mu}} \lambda_{\tilde{K}}(\tilde{\mu}),
$$

where the choices of evolution scales

$$
\mu_{0}:=\mu_{0}(\zeta)=\frac{\zeta P^{-}}{N}, \quad \mu_{1}:=\mu_{1}(\zeta)=e^{2} \zeta P^{-},
$$

are made to diminish the initial conditions $\tilde{K}^{(r)}\left(\mu_{0}\right)$ and $G^{(r)}\left(\mu_{1}\right)$, and the anomalous dimensions at one-loop level are determined as

$$
\lambda_{\tilde{K}}\left(\mu_{f}\right)=-G^{(r)}\left(\mu_{f}\right)=\frac{\alpha_{s}\left(\mu_{f}\right) C_{F}}{2 \pi} .
$$


The rapidity evolution equation of TMD pion wave function in Mellin and impact parameter spaces reads

$$
\zeta^{2} \frac{d}{d \zeta^{2}} \tilde{\Phi}\left(N, b, \zeta^{2}, \mu_{f}\right)=\tilde{\Gamma}\left(N, b, \zeta^{2}\right) \tilde{\Phi}\left(N, b, \zeta^{2}, \mu_{f}\right)
$$

with the evolution kernel

$$
\tilde{\Gamma}\left(N, b, \zeta^{2}\right)=\tilde{K}^{(r)}\left(\mu_{f}\right)+G^{(r)}\left(\mu_{f}\right)=-\int_{\mu_{0}(\zeta)}^{\mu_{1}(\zeta)} \frac{d \tilde{\mu}}{\tilde{\mu}} \lambda_{K}(\tilde{\mu}) .
$$

The factorization scale evolution can be written as

$$
\mu_{f} \frac{d}{d \mu_{f}} \tilde{\Phi}\left(N, b, \zeta^{2}, \mu_{f}\right)=\frac{3}{2} \frac{\alpha_{s}\left(\mu_{f}\right) C_{F}}{\pi} \tilde{\Phi}\left(N, b, \zeta^{2}, \mu_{f}\right),
$$

Solving the rapidity and scale evolution equations yields

$$
\begin{aligned}
\tilde{\Phi}\left(N, b, \zeta^{2}, \mu_{f}\right)=\exp \{ & -\int_{\zeta_{0}^{2}}^{\zeta^{2}} \frac{d \tilde{\zeta}^{2}}{\tilde{\zeta}^{2}}\left[\int_{\mu_{0}(\tilde{\zeta})}^{\mu_{1}(\tilde{\zeta})} \frac{d \tilde{\mu}}{\tilde{\mu}} \lambda_{K}(\tilde{\mu}) \theta\left(\mu_{1}(\tilde{\zeta})-\mu_{0}(\tilde{\zeta})\right)\right] \\
& \left.+\frac{3}{2} \int_{\mu_{i}}^{\mu_{f}} \frac{d \tilde{\mu}}{\tilde{\mu}} \frac{\alpha_{s}(\tilde{\mu}) C_{F}}{\pi}\right\} \tilde{\Phi}\left(N, b, \zeta_{0}^{2}, \mu_{i}\right) .
\end{aligned}
$$

Applying the fact that a physical quantity is free of rapidity and scale dependencies one can easily derive the joint resummation improved hard function. Putting all the pieces together, one can finally arrive at the resummation and RG improved $\gamma^{*} \pi \rightarrow \gamma$ form factor

$$
\begin{aligned}
F\left(Q^{2}\right)= & \exp \left\{-\int_{\zeta_{0}^{2}}^{\zeta_{1}^{2}} \frac{d \tilde{\zeta}^{2}}{\tilde{\zeta}^{2}}\left[\int_{\mu_{0}(\tilde{\zeta})}^{\mu_{1}(\tilde{\zeta})} \frac{d \tilde{\mu}}{\tilde{\mu}} \lambda_{K}(\tilde{\mu}) \theta\left(\mu_{1}(\tilde{\zeta})-\mu_{0}(\tilde{\zeta})\right)\right]\right. \\
& \left.+\frac{3}{2} \int_{\mu_{i}}^{t} \frac{d \tilde{\mu}}{\tilde{\mu}} \frac{\alpha_{s}(\tilde{\mu}) C_{F}}{\pi}\right\} \tilde{\Phi}\left(N, b, \zeta_{0}^{2}, \mu_{i}\right) \otimes \tilde{H}\left(N, b, \zeta_{1}^{2}, Q^{2}, t\right) \\
\equiv & \tilde{\Phi}\left(N, b, \zeta_{1}^{2}, t\right) \otimes \tilde{H}\left(N, b, \zeta_{1}^{2}, Q^{2}, t\right) .
\end{aligned}
$$

\section{Joint-resummation Improved Pion Transition form Factor}

To transform back into the momentum space, one needs to perform the inverse Mellin transformation

$$
\bar{\Phi}\left(x, b, \zeta_{1}^{2}, t\right)=\int_{c-i \infty}^{c+i \infty} \frac{d N}{2 \pi i}(1-x)^{-N} \tilde{\Phi}\left(N, b, \zeta_{1}^{2}, t\right),
$$

with the initial condition of TMD wave function which is assumed to be

$$
\Phi\left(x, k_{T}, \zeta_{0}^{2}, \mu_{i}\right)=\phi\left(x, \zeta_{0}^{2}, \mu_{i}\right) \Sigma\left(k_{T}^{2}\right) .
$$

The transverse-momentum dependence is taken as

$$
\Sigma\left(k_{T}^{2}\right)=4 \pi \beta^{2} \exp \left(-\beta^{2} k_{T}^{2}\right),
$$



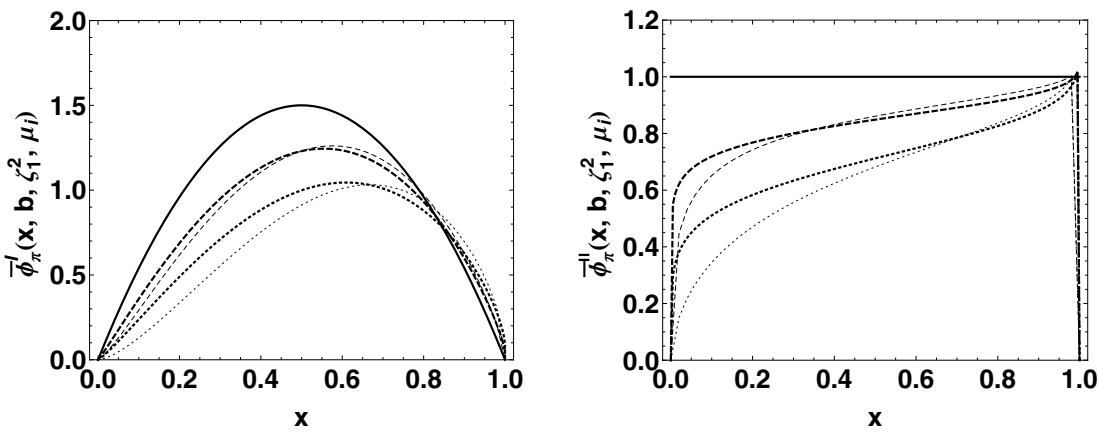

Fig. 2. Shape of the pion wave function with different models shown in Eq. (24). (a) the solid, (thin) dashed and (thin) dotted curves correspond to the initial condition $\phi^{\mathrm{I}}\left(x, \zeta_{0}^{2}, \mu_{i}\right)$, the jointresummation improved wave function $\bar{\Phi}^{\mathrm{I}}\left(x, \frac{2 \tilde{a}}{a P^{-}}, \zeta_{1}^{2}, \mu_{i}\right)$ and $\bar{\Phi}^{\mathrm{I}}\left(x, \frac{4 \tilde{a}}{a P^{-}}, \zeta_{1}^{2}, \mu_{i}\right)$ for a frozen $\alpha_{s}=0.3$ (running $\alpha_{s}$ ). (b) the same for the flat pion wave function $\bar{\Phi}^{\mathrm{II}}\left(x, b, \zeta_{1}^{2}, \mu_{i}\right)$.

where the shape parameter $\beta$ is determined by the mean transverse momentum of pion, and the two models for the longitudinal distributions discussed here are

$$
\phi^{\mathrm{I}}\left(x, \zeta_{0}^{2}, \mu_{i}\right)=6 x(1-x), \quad \phi^{\mathrm{II}}\left(x, \zeta_{0}^{2}, \mu_{i}\right)=1 .
$$

The inverse Mellin transformation is firstly worked out with a frozen strong coupling $\alpha_{s}$ to make the analytical structure of resummation improved wave function more transparent and then with a running $\alpha_{s}$. The actual effect of rapidity evolution with different initial conditions can be understood from figure 2. One can observe that the joint resummation can generate stronger suppression for the small $x$ region compared to the moderate $x$ region, independent of the initial conditions. In particular, such end-point suppression strengthens with the impact parameter $b$ for a given longitudinal momentum fraction $x$. Switching on the running of strong coupling, the resummation effect turns out to be similar to that for a frozen coupling, except that the end-point suppression becomes even stronger. These observations indicate that the soft-gluon exchange effects will not play a significant role taking into account of the joint resummation effect, which further boosts our confidence on the applicability of TMD factorization in hard exclusive processes at moderate momentum transfer.

The pion transition form factor responsible for $\gamma^{*} \pi^{0} \rightarrow \gamma$ process is defined as

$$
\left\langle\gamma\left(P^{\prime}, \epsilon^{*}\right)\left|j_{\mu}^{e m}(q)\right| \pi^{0}(P)\right\rangle=i g_{e m}^{2} \varepsilon_{\mu \nu \alpha \beta} \epsilon^{* \nu} P^{\alpha} P^{\prime \beta} F\left(Q^{2}\right),
$$

where $j_{\mu}^{e m}(q)$ is an electromagnetic current. The new TMD factorization formula of pion transition form factor including joint resummation reads

$$
\begin{aligned}
F\left(Q^{2}\right)= & \frac{\sqrt{2} f_{\pi}}{3} \int_{0}^{1} d x \int_{0}^{\infty} b d b \bar{\Phi}\left(x, b, \zeta_{1}^{2}, t\right) K_{0}(\sqrt{x} Q b) \\
& \times\left[1-\frac{\alpha_{s}(t) C_{F}}{4 \pi}\left(3 \ln \frac{t^{2} b}{2 \sqrt{x} Q}+\ln 2+2\right)\right],
\end{aligned}
$$




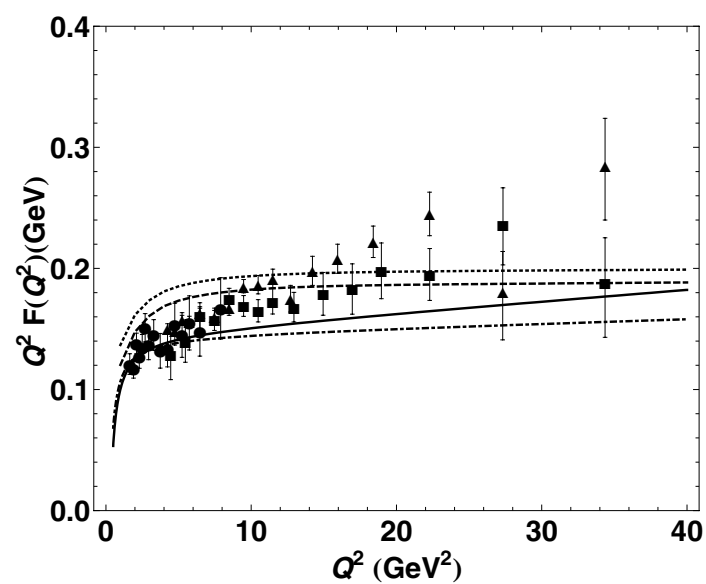

Fig. 3. Pion transition form factor computed from the asymptotic model of pion wave function. The dashed and dotted (dot-dashed and solid) curves indicate the LO and NLO predictions from the conventional resummations (joint resummation). The experimental data are from CLEO (dots), BaBar (triangles), and Belle (squares).

where the hard scale $t=\max (\sqrt{x} Q, 1 / b)$ is set to be the typical quantum fluctuation of the considered scattering process.

The computed pion transition form factor $Q^{2} F\left(Q^{2}\right)$ with the asymptotic pion wave function is displayed in figure 3 . One can observe that the computed form factor saturates rapidly for $Q^{2}>5 \mathrm{GeV}^{2}$ with the conventional resummations at both leading-order (LO) and NLO levels. The asymptotic pion wave function can result in the predictions in reasonable agreement with the Belle data, ${ }^{19}$ however, it fails to accommodate the CLEO measurements ${ }^{20}$ and BaBar data ${ }^{1}$ in both small and large $Q^{2}$ region. The joint-resummation effect is found to decrease the LO and NLO predictions in the conventional approach by $(11-16) \%$ and $(8-27) \%$, due to the stronger suppression at the small $x$ which is the dominant region contributed to the pion transition form factor. The failure of describing the experimental data suggests that the pion wave function might be broader than that predicted by perturbative QCD in the asymptotic limit. Fitting the experimental data requires that the second Gegenbauer coefficient of pion wave function $a_{2}=0.05$ which is significantly smaller than that extracted from the light-cone sum rule calculation of pion electromagnetic form factor. ${ }^{21}$ It is then evident that the predictions with flat pion wave function will overshot the data in TMD factorization. It needs to point out that the Gegenbauer moments in TMD wave function are not necessarily the same as that in the LCDA.

\section{Conclusion}

To summarize, an evolution equation of TMD pion wave function is constructed to organize the mixed logarithm $\ln x \ln \left(\zeta_{P}^{2} / k_{T}^{2}\right)$ applying the joint resummation 
technique. The crucial consequence of joint resummation lies in the stronger suppression of small $x$ region enhancing the applicability of perturbative QCD factorization in hard exclusive processes at moderate momentum transfer. Confronting the predictions of pion transition form factor from TMD factorization with experimental data reveals a broader pion structure than that expected in the asymptotic limit. It is natural to expect that the resummation approach developed in Ref. [12] can be extended to more sophisticated hard reactions such as pion electromagnetic form factor and $B \rightarrow \pi$ form factors.

\section{Acknowledgments}

I would like to thank the organizers for inviting me to this enjoyable workshop. This research is supported by the DFG-Sonderforschungsbereich/Transregio 9 "Computergestützte Theoretische Teilchenphysik".

\section{References}

1. B. Aubert et al. [BaBar Collaboration], Phys. Rev. D 80, 052002 (2009).

2. S. S. Agaev, V. M. Braun, N. Offen and F. A. Porkert, Phys. Rev. D 83054020 (2011); Phys. Rev. D 86, 077504 (2012).

3. A. V. Radyushkin, Phys. Rev. D 80094009 (2009).

4. H. n. Li and S. Mishima, Phys. Rev. D 80, 074024 (2009).

5. S. Nandi and H. n. Li, Phys. Rev. D 76, 034008 (2007).

6. H. n. Li, Y. L. Shen, Y. M. Wang and H. Zou, Phys. Rev. D 83, 054029 (2011).

7. S. Cheng, Y. Y. Fan and Z. J. Xiao, Phys. Rev. D 89, 054015 (2014).

8. H. n. Li, Y. L. Shen and Y. M. Wang, Phys. Rev. D 85, 074004 (2012); JHEP 1302, 008 (2013).

9. S. Cheng, Y. Y. Fan, X. Yu, C. D. L and Z. J. Xiao, Phys. Rev. D 89, 094004 (2014).

10. Y. L. Shen and Y. M. Wang, arXiv:1409.1048 [hep-ph].

11. H. n. Li, Phys. Lett. B 454, 328 (1999).

12. H. N. Li, Y. L. Shen and Y. M. Wang, JHEP 1401, 004 (2014).

13. J. C. Collins, Acta Phys. Polon. B 34, 3103 (2003).

14. J. Collins, Int. J. Mod. Phys. Conf. Ser. 4, 85 (2011).

15. A. Bacchetta, D. Boer, M. Diehl and P. J. Mulders, JHEP 0808, 023 (2008).

16. J. Collins, Foundations of perturbative QCD, (Cambridge monographs on particle physics, nuclear physics and cosmology, 32).

17. H. n. Li and Y. M. Wang, Transverse-momentum dependent pion wave function, to appear soon.

18. J. C. Collins and D. E. Soper, Nucl. Phys. B 193, 381 (1981) [Erratum-ibid. B 213, $545(1983)]$.

19. S. Uehara et al. [Belle Collaboration], Phys. Rev. D 86, 092007 (2012).

20. J. Gronberg et al. [CLEO Collaboration], Phys. Rev. D 5733 (1998).

21. A. Khodjamirian, T. Mannel, N. Offen and Y.-M. Wang,

Phys. Rev. D 83, 094031 (2011). 